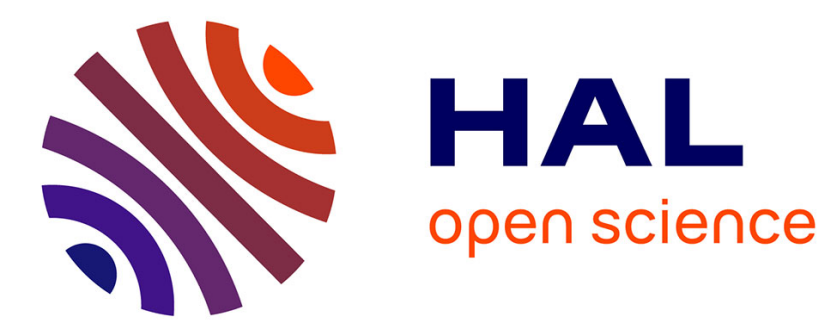

\title{
Consensus Reaching With Heterogeneous User Preferences
}

Hélène Le Cadre, Enrique Rivero Puente, Hanspeter Höschle

\section{To cite this version:}

Hélène Le Cadre, Enrique Rivero Puente, Hanspeter Höschle. Consensus Reaching With Heterogeneous User Preferences. 2019. hal-01874798v2

\section{HAL Id: hal-01874798 \\ https://hal.science/hal-01874798v2}

Preprint submitted on 4 Mar 2019

HAL is a multi-disciplinary open access archive for the deposit and dissemination of scientific research documents, whether they are published or not. The documents may come from teaching and research institutions in France or abroad, or from public or private research centers.
L'archive ouverte pluridisciplinaire HAL, est destinée au dépôt et à la diffusion de documents scientifiques de niveau recherche, publiés ou non, émanant des établissements d'enseignement et de recherche français ou étrangers, des laboratoires publics ou privés. 


\title{
Consensus Reaching With Heterogeneous User Preferences
}

\author{
Hélène Le Cadre, Enrique Rivero Puente, and Hanspeter Höschle \\ VITO/EnergyVille, Thor Scientific Park, 3600 Genk, Belgium \\ helene.lecadre@energyville.be
}

\begin{abstract}
In this paper, we consider consumers and prosumers who interact on a platform. Consumers buy energy to the platform to maximize their usage benefit while minimizing the cost paid to the platform. Prosumers, who have the possibility to generate energy, self-consume part of it to maximize their usage benefit and sell the rest to the platform to maximize their revenue. Product differentiation is introduced and consumers can specify preferences regarding locality, RES-based generation, and matchings with the prosumers. The consumers and prosumers' problems being coupled through a matching probability, we provide analytical characterizations of the resulting Nash equilibrium. Assuming supply-shortages occur, we reformulate the platform problem as a consensus problem that we solve using Alternating Direction Method of Multipliers (ADMM), enabling minimal information exchanges between the nodes. On top of the platform, a trust-based mechanism combining exploitation of nodes with good reputation and exploration of new nodes, is implemented to determine the miner node which validates the transactions. A case study is provided to analyze the impact of preferences and miner selection dynamic process.
\end{abstract}

Keywords: Game theory · Two-sided market · Local community · ADMM.

\section{Introduction}

The increasing amount of Distributed Energy Resources (DERs), which have recently been integrated in power systems, the development of new storage technologies, and the more proactive role of consumers (prosumers) have transformed the classical centralized power system operation (mostly based on unit commitment) by introducing more uncertainty and decentralization in the decisions. Following this trend, electricity markets are starting to restructure, from a centralized market design in which all the operations were managed by a global (central) market operator, modeled as a classical constrained optimization problem, to more decentralized designs involving local energy communities which can trade energy by the intermediate of the global market operator [6] or, in a peerto-peer setting [8]. Coordinating local renewable energy sources (RES)-based generators to satisfy the demand of local energy communities, could provide significant value to the power systems, by decreasing the need for investments 
in conventional generations and transmission networks. In practice, the radial structure of the distribution grid calls for hierarchical market designs, involving transmission and distribution network operators [7]. But, various degrees of coordination can be envisaged, from full coordination organized by a global market operator (transmission network operator), to bilateral contract networks, to fully decentralized market designs allowing peer-to-peer energy trading between the prosumers in a distributed fashion [8], or within and between coalitions of prosumers [12].

In the energy sector, peer-to-peer energy trading is a novel paradigm of power system operation, where prosumers providing their own energy from solar panels, storage technologies, demand response mechanisms, exchange energy/capacity with one another. Zhang et al. provide in [15] an exhaustive list of projects and trails all around the world, which build on new innovative approaches for peer-topeer energy trading. A large part of these projects rely on platforms which match RES-based generators and consumers according to their preferences and locality aspects (such as Piclo in the UK, TransActive Grid in Brooklyn, US, Vandebron in the Netherlands, etc.). In the same vein, cloud-based virtual market places to deal with excess generation within microgrids are developed by PeerEnergyCloud and Smart Watts in Germany. Some other projects rely on local community building for investment sharing in batteries, solar PV panels, etc., in exchange of bill reduction or to obtain a certain level of autonomy with respect to the global grid (such as Yeloha and Mosaic in the US, SonnenCommunity in Germany, etc.).

Platform design is an active area of research in the two-sided market economics literature [3]. Three needs are identified for platform deployment: a first requirement is to help buyers and sellers find each other, taking into account preference heterogeneity. This requires to find a trade-off between low-entry cost and information retrieval from big, heterogeneous, and dynamic information flows. Buyers and sellers search can be performed in a centralized fashion (Amazon, Uber), or it might allow for effective decentralized search (Airbnb, eBay), or even fully distributed search (OpenBazaar, Arcade City). A second need is to set prices that balance demand and supply, and ensure that prices are set competitively in a decentralized fashion. A third requirement is to maintain trust in the market, relying on reputation and feedback mechanisms. Sometimes, supply might be insufficient and subsidies should be designed to encourage sharing on the platform [3].

From an information and communication technology (ICT) perspective, a fully decentralized market design provides a robust framework since if one node in a local market is attacked or in case of failures, the whole architecture should remain in place and information could find other paths to circulate from one point to another, avoiding malicious nodes/corrupted paths. From an algorithmic point of view, such a setting enables the implementation of algorithms that preserve privacy of the local market agents (requiring from them to not share more than their dual variables - e.g., local prices - updates). This also creates high computational challenges, especially if the number of local markets/peers 
is high. Trust, security, and transparency issues for peer-to-peer energy markets could rely on the emergence of blockchain technology. A blockchain is a continuously growing list of records, called blocks, which are linked and secured using cryptography. Each block typically contains a hash pointer as a link to a previous block, a time-stamp and transaction data. By design, blockchains are inherently resistant to modification of the data. A blockchain can serve as "an open, distributed ledger that can record transactions between two or more parties efficiently and in a verifiable and permanent way." For use as a distributed ledger, a blockchain is typically managed by a peer-to-peer network collectively adhering to a protocol for validating new blocks. Once recorded, the data in any given block cannot be altered retroactively without the alteration of all subsequent blocks, which needs a collusion of the network majority. The most important function about the records (called transactions in the literature) is their traceability. For each record, it is possible to trace its origin and by whom it has been created and/or exchanged. The verification of the correctness of each transaction could be done by every participant of the chain. However, there is a specific role for creating a block at every time period and thus, guaranteeing that the transactions within it are correct. This is the role of the so-called miner, who provides computational power to check the transactions and put them together to form blocks, in exchange for a fee [13]. On top of blockchain technology, smart contracts are autonomous computer systems, written in code, that manage executions in the form of rules between parties on the Blockchain. For example, the reaching of a consensus between nodes, specific events (train and airplane delays, conditions for a contract to hold) can be detected online, and the execution of the smart contract is automatically triggered [11].

To avoid any influence of a malicious node, consensus algorithms are employed. Bitcoin, the first existing blockchain technology, relies on Proof-of-Work (PoW): nodes have to solve a mathematical problem (puzzle) so complex that the only way to solve it is to try every possible permutation. This results in a slow and excessively energy-greedy program. Proof-of-Stake (PoS) used by Etherum, another well-known blockchain technology, is a method for consensusbuilding between the nodes, in which a miner is randomly selected based on its wealth instead of reputation. However, the miner node selection seems rather arbitray and not relying on trust. In [9], Munsing et al. consider a large-scale load scheduling problem that they decompose with ADMM [1], [4]. Consensus is reached when ADMM converges to a stable solution; payments and penalties are then computed based on the ADMM outcome. ADMM-based consensus problems consist in computing the optimum of a (large-scale) optimization problem, where nodes exchange information only with a subset of the other nodes. Several algorithms for consensus can be found in the literature and have attracted much attention in the last decades in the broader framework of sensor management and data fusion: they differentiate on the basis of the amount of communication and computation they use, on their scalability with respect to the number of nodes, on their (online) adaptability, and, finally, they can be deterministic or randomized [2]. 
The contributions of this manuscript can be summarized as follows:

(a) We formulate an equilibrium problem representing a two-sided local market platform with consumers and prosumers. Consumers can have preferences toward characteristics of the electricity products (RES-based generation, locality) and matchings with the prosumers.

(b) We discuss different algorithms to compute the outcome of such a market platform and highlight differences in the need of sharing information with the local market operator and other participants.

(c) We propose a method on how the role of the market operator could be organized with smart contracts and the miner node can be selected among the market participants relying on a trust-based mechanism.

(d) We illustrate (b) and (c) based on a small case study highlighting the effect of certain parameter, most importantly, the consumer preferences, and the tuning of the parameters for the trust-based mechanism.

The remainder of the paper is structured as follows. Section 2 introduces the mathematical description of the market platform and its participants. The proposed ADMM algorithm to compute the clearing of the market platform is introduced in Section 3. Section 4 illustrates and discusses several elements of the market platform in a case study. Conclusions of the paper are drawn in Section 5 .

\section{Model Description}

We consider a set $\mathcal{N}$ of $N$ nodes. Each node can be either a prosumer $P$ having the possibility to generate and consume (part) of her own energy while selling the excess by the intermediate of a sharing platform operated by a local Market Operator (MO), or a consumer-only $C$ without generation facility. We denote by $\mathcal{P}$, the prosumer set, and by $\mathcal{C}$, the consumer-only set. Furthermore, we have the relations: $\mathcal{P} \cup \mathcal{C}=\mathcal{N}$ and $\mathcal{C} \cap \mathcal{N}=\emptyset$. Local energy demand and supply balance is guaranteed by the local MO, who can sell excess production or buy shortage to the power grid.

Our inspiration for the prosumer-consumer interaction model comes for the literature of two-sided markets [3], though the structure of electricity markets and asymmetry of prosumer role, who can benefit from consumption of selfproduction (therefore, becoming consumers) and excess production selling by the intermediate of the sharing platform (therefore, becoming producers), makes extensions of this literature tricky. The consumer-prosumer platform framework is visualized in Fig. 1.

\subsection{Modeling Consumers}

For each consumer $C \in \mathcal{C}$, we denote the usage benefit obtained from consuming a quantity $y_{t}^{C}$ of energy, by $U_{C}\left(y_{t}^{C}\right)$. We assume that $U_{C}($.$) is only known to$ the consumer and is not public knowledge. We make the assumption that $U_{C}($. 


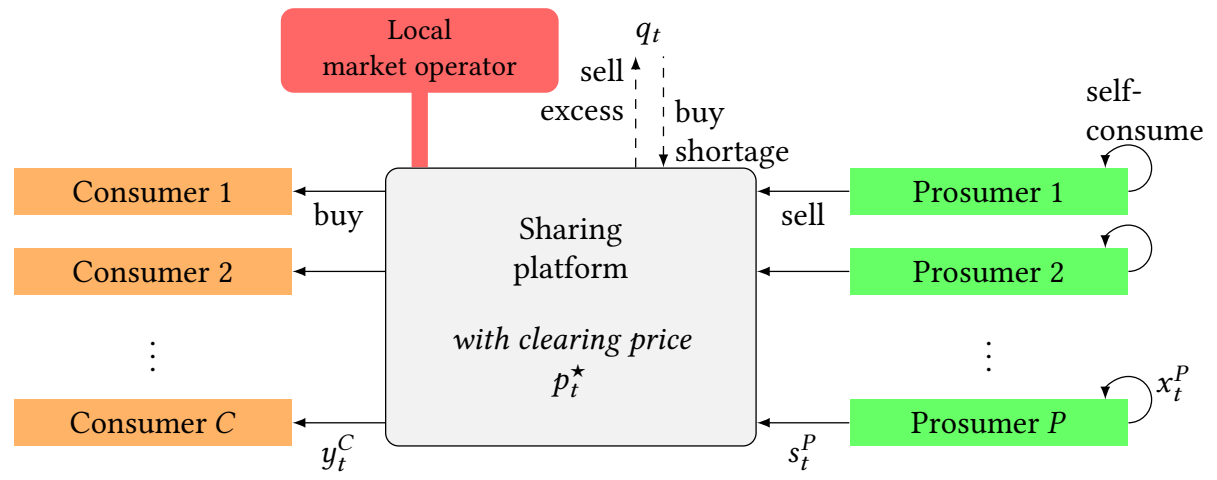

Fig. 1: Example of a sharing platform involving consumers-only on one side and prosumers on the other side.

is continuous and strictly concave and non-negative on $\mathbb{R}_{+}$. To fix the idea, we assume that consumer $C$ usage benefit is a quadratic function of the consumer demand $y_{t}^{C}$, leading to the following definition:

$$
U_{C}\left(y_{t}^{C}\right)=-\eta^{C}\left(y_{t}^{C}-y_{t}^{C \sharp}\right)^{2}+\tilde{\eta}^{C},
$$

where $\eta^{C}, \tilde{\eta}^{C}$ are positive parameters, and $y_{t}^{C \sharp}$ is the target demand of consumer $C$ at time period $t$. For the usage benefit to remain non-negative on the interval of definition of $y_{t}^{C}$, we impose conditions on the parameters such that $U_{C}(0) \geq 0$ and $U_{C}\left(\kappa^{C}\right) \geq 0$, leading to $\kappa^{C}-\sqrt{\frac{\tilde{\eta}^{C}}{\eta^{C}}} \leq y_{t}^{C \sharp} \leq \sqrt{\frac{\tilde{\eta}^{C}}{\eta^{C}}}, \forall t$. Note that the maximum usage benefit is reached in $U_{C}\left(y_{t}^{C \sharp}\right)=\tilde{\eta}^{C}$ and in case $U_{C}(0)=0$, i.e., zero demand implies zero usage benefit, we have the following relation between the consumer target demand and usage benefit parameters: $\eta^{C}=\frac{U_{C}\left(y_{t}^{\mathrm{C}}\right)}{\left(y_{t}^{C^{\sharp}}\right)^{2}}$.

We refine the consumer model by introducing product differentiation [8]. To that purpose, we first assume that consumer $C$ defines the percentages $\xi_{\mathrm{RES}}^{C}$, $\xi_{\text {Loc }}^{C} \in[0 ; 1]$ of his target demand that comes from "RES-based generation" and local energy prosumers, where the "locality" of prosumer $P$ with respect to consumer $C$ is measured by the distance between $P$ and $C^{1}$. So, we assume that some consumers might prefer to be served by prosumers in a local area, e.g., within a limited radius. This means that the percentage of consumer $C$ target demand coming from RES-based generation and local prosumers is defined as $\xi_{\mathrm{RES}}^{C} y_{t}^{C \sharp}$ and $\xi_{\mathrm{Loc}}^{C} y_{t}^{C \sharp}$ respectively. Note that we do not impose that $\xi_{\mathrm{RES}}^{C}+\xi_{\mathrm{Loc}}^{C}=1$ because "RES-based generation" and "locality" are not mutually exclusive preferences, meaning that some "green" consumers might want to cover their demand with 100\% RES-based generation and local production only. We add a second level of complexity, by assuming that consumer $C$ has in-

${ }^{1}$ Note that the preference model is generic enough to introduce other levels of product differentiation. 
trinsic preferences with respect to the prosumers in $\mathcal{P}$ (that we will call later on "matching preferences"), which lead them to define how they ideally wish to split their demand between the prosumers. Let $\alpha_{\mathrm{RES}}^{C P}$ and $\alpha_{\text {Loc }}^{C P}$ be positive parameters associated with any prosumer $P \in \mathcal{P}$ production, characterizing the preferences of the consumer $C$ regarding his matching with the prosumers on the platform, and such that $\frac{1}{\operatorname{card}(\mathcal{P}) \xi_{\mathrm{RES}}^{C}} \sum_{P \in \mathcal{P}} \alpha_{\mathrm{RES}}^{C P}=\frac{1}{\operatorname{card}(\mathcal{P}) \xi_{\mathrm{Loc}}^{C}} \sum_{P \in \mathcal{P}} \alpha_{\mathrm{Loc}}^{C P}=1, \forall C \in \mathcal{C}$.

Extending Equation (1) to the RES-based generation and local production usage, we define the usage benefit resulting from RES-based and local consumption as follows:

$$
\begin{aligned}
U_{C}^{\mathrm{Loc}}\left(y_{t}^{C}\right) & =-\eta_{\mathrm{Loc}}^{C}\left(\sum_{P \in \mathcal{P}} \alpha_{\mathrm{Loc}}^{C P} y_{t}^{C}-\xi_{\mathrm{Loc}}^{C} y_{t}^{C \sharp}\right)^{2}+\tilde{\eta}_{\mathrm{Loc}}^{C} . \\
U_{C}^{\mathrm{RES}}\left(y_{t}^{C}\right) & =-\eta_{\mathrm{RES}}^{C}\left(\sum_{P \in \mathcal{P}} \alpha_{\mathrm{RES}}^{C P} y_{t}^{C}-\xi_{\mathrm{RES}}^{C} y_{t}^{C \sharp}\right)^{2}+\tilde{\eta}_{\mathrm{RES}}^{C} .
\end{aligned}
$$

We introduce $w_{\mathrm{Loc}}, w_{\mathrm{RES}}$, and $w_{0}$, as non-negative parameters characterizing the relative importance of locality and RES-based generation in the consumer total usage benefit, with respect to the no-product differentiation case. Then, consumer $C$ total usage benefit can be decomposed as the weighted sum of the benefits retrieved from local production and RES-based generation consumption, and usage benefit (1) without product differentiation ${ }^{2}$ :

$$
\tilde{U}_{C}\left(y_{t}^{C}\right)=w_{\mathrm{Loc}} U_{C}^{\mathrm{Loc}}\left(y_{t}^{C}\right)+w_{\mathrm{RES}} U_{C}^{\mathrm{RES}}\left(y_{t}^{C}\right)+w_{0} U_{C}\left(y_{t}^{C}\right) .
$$

The utility consumer $C$ obtains from energy consumption $y_{t}^{C}, \Pi_{C}\left(y_{t}^{C}\right)$, is given by the benefit $\tilde{U}_{C}($.$) minus the cost to buy energy on the platform operated$ by the local market operator (MO), $p_{t}^{\star}$ times the consumption $y_{t}^{C}$. Formally, we have:

$$
\Pi_{C}\left(y_{t}^{C}\right)=\tilde{U}_{C}\left(y_{t}^{C}\right)-p_{t}^{\star} y_{t}^{C} .
$$

Each consumer $C$ determines his demand $y_{t}^{C}$ so as to maximize the sum of his utility function (3) and potential mining fee, under non-negativity and maximum capacity of consumption $\kappa^{C}$ constraints:

$$
\begin{array}{rlr}
\max _{y_{t}^{C}} & \Pi_{C}\left(y_{t}^{C}\right), & \\
\text { s.t. } & y_{t}^{C} \leq \kappa^{C}, & \\
& 0 \leq y_{t}^{C} . & \left(\psi_{t}^{C}\right) \\
& & \left(\tilde{\psi}_{t}^{C}\right)
\end{array}
$$

We prove in the proposition below that there always exists a solution to the consumer utility maximization problem.

\footnotetext{
${ }^{2}$ Note that (1) is added in Equation (2) to counter-balance the effects of product differentiation that might encourage the consumer to excess her demand in case where the sum of the locality and RES-based generation demand targets is larger $\left(\xi_{\mathrm{RES}}^{C}+\xi_{\mathrm{Loc}}^{C}>1\right)$ than the actual demand target.
} 
Proposition 1. Consumer $C$ utility function $\Pi_{C}($.$) is strictly concave in his$ demand $y_{t}^{C}$ and maximized at a single optimum solution of optimization problem (4) under constraints (5), (6):

$$
\begin{aligned}
y_{t}^{C \star} & =\frac{\left(2 w_{0} \eta^{C}+2 w_{R E S} \eta_{R E S} \xi_{R E S}^{C}\left(\sum_{P \in \mathcal{P}} \alpha_{R E S}^{C P}\right)\right) y_{t}^{C \sharp}}{2 w_{0} \eta^{C}+2 w_{R E S} \eta_{R E S}^{C}\left(\sum_{P \in \mathcal{P}} \alpha_{R E S}^{C P}\right)^{2}+2 w_{L o c} \eta_{L o c}^{C}\left(\sum_{P \in \mathcal{P}} \alpha_{L o c}^{C P}\right)^{2}} \\
& +\frac{\left(2 w_{L o c} \eta_{L o c}^{C} \xi_{L o c}^{C}\left(\sum_{P \in \mathcal{P}} \alpha_{L o c}^{C P}\right)\right) y_{t}^{C \sharp}-p_{t}^{\star}-\left(\tilde{\Psi}_{t}^{C}-\Psi_{t}^{C}\right)}{2 w_{0} \eta^{C}+2 w_{R E S} \eta_{R E S}^{C}\left(\sum_{P \in \mathcal{P}} \alpha_{R E S}^{C P}\right)^{2}+2 w_{L o c} \eta_{L o c}^{C}\left(\sum_{P \in \mathcal{P}} \alpha_{L o c}^{C P}\right)^{2}},
\end{aligned}
$$

with $\Psi_{t}^{C}\left(y_{t}^{C \star}-\kappa^{C}\right)=0, \tilde{\Psi}_{t}^{C} y_{t}^{C \star}=0, \Psi_{t}^{C} \geq 0, \tilde{\Psi}_{t}^{C} \geq 0$.

Proof. The Lagrangian function associated with optimization problem (4)-(6) writes down as follows: $\mathcal{L}_{C}\left(y_{t}^{C}, \Psi_{t}^{C}, \tilde{\Psi}_{t}^{C}\right)=\Pi_{C}\left(y_{t}^{C}\right)-\Psi_{t}^{C}\left(y_{t}^{C}-\kappa^{C}\right)+\tilde{\Psi}_{t}^{C} y_{t}^{C}$. Complementarity slackness conditions take the form: $\Psi_{t}^{C}\left(y_{t}^{C}-\kappa^{C}\right)=0, \tilde{\Psi}_{t}^{C} y_{t}^{C}=$ 0 , and the dual feasibility constraints impose that: $\Psi_{t}^{C} \geq 0, \tilde{\Psi}_{t}^{C} \geq 0$.

Derivating the Lagrangian function with respect to $y_{t}^{C}$, we obtain:

$$
\begin{aligned}
& \frac{\partial \mathcal{L}_{C}\left(y_{t}^{C}, \Psi_{t}^{C}, \tilde{\Psi}_{t}^{C}\right)}{\partial y_{t}^{C}}=-2 w_{0} \eta^{C}\left(y_{t}^{C}-y_{t}^{C \sharp}\right)-2 w_{\mathrm{RES}} \eta_{R E S}^{C}\left(\sum_{P \in \mathcal{P}} \alpha_{\mathrm{RES}}^{C P} y_{t}^{C}-\xi_{\mathrm{RES}}^{C} y_{t}^{C \sharp}\right) \\
& \left(\sum_{P \in \mathcal{P}} \alpha_{\mathrm{RES}}^{C P}\right)-2 w_{\mathrm{Loc}} \eta_{\text {Loc }}^{C}\left(\sum_{P \in \mathcal{P}} \alpha_{\mathrm{Loc}}^{C P} y_{t}^{C}-\xi_{\mathrm{Loc}}^{C} y_{t}^{C \sharp}\right)\left(\sum_{P \in \mathcal{P}} \alpha_{\mathrm{Loc}}^{C P}\right)-p_{t}^{\star}-\Psi_{t}^{C}+\tilde{\Psi}_{t}^{C} .
\end{aligned}
$$

Derivating the Lagrangian function twice with respect to $y_{t}^{C}$, we obtain:

$$
-2 w_{0} \eta^{C}-2 w_{\mathrm{RES}} \eta_{R E S}^{C}\left(\sum_{P \in \mathcal{P}} \alpha_{\mathrm{RES}}^{C P}\right)^{2}-2 w_{\mathrm{Loc}} \eta_{\text {Loc }}^{C}\left(\sum_{P \in \mathcal{P}} \alpha_{\mathrm{Loc}}^{C P}\right)^{2}<0 .
$$

This implies that $\Pi_{C}($.$) is strictly concave in y_{t}^{C}$. Therefore, it admits a unique optimum. At the optimum in $y_{t}^{C \star},\left.\frac{\partial \mathcal{L}_{C}\left(y_{t}^{C}, \Psi^{C}, \tilde{\Psi}^{C}\right)}{\partial y_{t}^{C}}\right|_{y_{t}^{C}=y_{t}^{C \star}}=0$, which is equivalent to $(7)$.

\subsection{Modeling Prosumers}

Prosumers have two ways to derive benefits from their production: using it themselves or selling it through the sharing platform by the intermediate of the local MO. We let $x_{t}^{P}$ be prosumer $P$ self-usage quantity and $s_{t}^{P}$ be the quantity of energy that prosumer $P$ shares through the platform. When prosumers consume their own energy production, they experience benefit from the consumption, like consumers-only. But, unlike consumers-only, they do not have to pay the local $\mathrm{MO}$ for their consumption, though their consumption may lead to production costs that can be interpreted as usage (in case of micro-CHP activation for example) or maintenance cost, or government taxes, etc. We denote the benefit from self-usage by $U_{P}\left(x_{t}^{P}\right)$ and the production cost incurred by $c_{P}\left(x_{t}^{P}+s_{t}^{P}\right)$. As in the case of the consumers-only, we assume that $U_{P}($.$) is continuous and$ 
strictly concave and non-negative on $\mathbb{R}_{+}$. In the same spirit as the consumer model, we assume that prosumer $P$ usage benefit is a quadratic function of the prosumer self-consumption $x_{t}^{P}$, leading to the following definition:

$$
U_{P}\left(x_{t}^{P}\right)=-\eta^{P}\left(x_{t}^{P}-x_{t}^{P \sharp}\right)^{2}+\tilde{\eta}^{P},
$$

where $\eta^{P}, \tilde{\eta}^{P}$ are non-negative parameters, and $x_{t}^{P \sharp}$ is the target self-consumption of prosumer $P$ at time period $t$. For the self-consumption benefit to remain nonnegative on the interval of definition of $x_{t}^{P}$, we impose conditions on the parameters such that $U_{P}(0) \geq 0$ and $U_{P}\left(\kappa^{P}\right) \geq 0$, leading to $\kappa^{P}-\sqrt{\frac{\tilde{\eta}^{P}}{\eta^{P}}} \leq x_{t}^{P \sharp} \leq$ $\sqrt{\frac{\tilde{\eta}^{P}}{\eta^{P}}}, \forall t$.

When the prosumers share their excess production through the platform, they receive a revenue and incur costs. The revenue they receive from sharing depends on how many other prosumers are also sharing their excess production. We introduce the probability $\mu\left(\boldsymbol{y}_{t}, \boldsymbol{s}_{t}\right)$ that a prosumer is matched to a consumeronly as follows:

$$
\mu\left(\boldsymbol{y}_{t}, \boldsymbol{s}_{t}\right):=\min \left\{\frac{\sum_{C \in \mathcal{C}} y_{t}^{C}}{\sum_{P \in \mathcal{P}} s_{t}^{P}} ; 1\right\}
$$

Naturally, $\mu\left(\boldsymbol{y}_{t}, \boldsymbol{s}_{t}\right)<1$ if, and only if, $\sum_{C \in \mathcal{C}} y_{t}^{C}<\sum_{P \in \mathcal{P}} s_{t}^{P}$, i.e., there is an excess of supply compared to the actual demand on the platform. And, $\mu\left(\boldsymbol{y}_{t}, \boldsymbol{s}_{t}\right)=1$ in case the consumer total demand is larger than the prosumers supply, therefore requiring that the local MO buys the missing quantity to the grid. In the following, for the sake of simplicity, we will write: $\mu_{t}:=\mu\left(\boldsymbol{y}_{t}, \boldsymbol{s}_{t}\right)$.

The utility function of a prosumer is the sum of the benefit she derives from the consumption of her self-production plus the expected revenue she derives from the selling of her excess production conditionally to her matching with a consumer minus her production cost, leading to the following mathematical expression:

$$
\Pi_{P}\left(x_{t}^{P}, \boldsymbol{y}_{t}, \boldsymbol{s}_{t}\right)=U_{p}\left(x_{t}^{P}\right)+p_{t}^{\star} \mu_{t} s_{t}^{P}-c_{P}\left(x_{t}^{P}+s_{t}^{P}\right) .
$$

Assuming that prosumer $P$ cost function is quadratic in her production, we set $c_{P}(x)=c_{P 2} x^{2}+c_{P 1} x+c_{P 0}, \forall x \in \mathbb{R}$ with $c_{P 2}, c_{P 1}, c_{P 0}$ non-negative parameters.

Each prosumer $P$ determines sharing and self-use variables $s_{t}^{P}$ and $x_{t}^{P}$ that maximize the sum of her utility function (10) and potential mining fee, under maximum capacity of production $\kappa^{P}$ constraint, by solving the following optimization problem:

$$
\begin{array}{rlr}
\max _{x_{t}^{P}, s_{t}^{P}} & \Pi_{P}\left(x_{t}^{P}, \boldsymbol{y}_{t}, \boldsymbol{s}_{t}\right), & \\
\text { s.t. } & x_{t}^{P}+s_{t}^{P} \leq \kappa^{P}, & \\
& 0 \leq x_{t}^{P}, s_{t}^{P} . & \left(\tilde{\Psi}_{t}^{P}\right) \\
& &
\end{array}
$$


Proposition 2. - If $\mu_{t}=1$, prosumer $P$ utility function is strictly concave in $x_{t}^{P}, s_{t}^{P}$ and maximized at a single optimum solution of optimization problem (11) under constraints (12), (13).

- If $\mu_{t}<1$, the Nash equilibrium solution of the non-cooperative game is uniquely defined as a parametric function of $\mu_{t}$, which can be obtained as solution of a fixed point equation.

In both cases, we have the relations: $\Psi_{t}^{P}\left(x_{t}^{P \star}+s_{t}^{P \star}-\kappa^{P}\right)=0, \tilde{\Psi}_{t}^{P} x_{t}^{P \star}=$ $\tilde{\Psi}_{t}^{P S} s_{t}^{P \star}=0, \Psi_{t}^{P} \geq 0, \tilde{\Psi}_{t}^{P} \geq 0, \tilde{\Psi}_{t}^{P S} \geq 0$.

Proof. Due to the space limit, the proof has been removed but can be found online at https://hal.archives-ouvertes.fr/hal-01874798/document

Proposition 3. Suppose that at the optimum $x_{t}^{P \star}>0, s_{t}^{P \star}>0, \forall P \in \mathcal{P}, \forall t$ and $\boldsymbol{y}_{t}^{\star}>0, \forall t$. There exists a market clearing price upper-bound $\bar{p}$, below which supply-shortages occur on the platform.

Proof. The proof can be found online at https://hal.archives-ouvertes.fr/ hal-01874798/document

Proposition 3 coincides with the results obtained in [3] for Didi Chuxing, the largest ridesharing platform in China: if the platform market clearing price is not high enough, suppliers might lack incentives to share their production on the platform and consumer-shortages might happen. In such cases, optimal design of subsidies might be necessary to give incentives to suppliers (prosumers) to share their supply.

\subsection{Modeling of Exchange of Market Platform}

In addition to consumers and prosumers, the market platform is assumed to be connected with a surrounding market environment (grid). An example would be the wholesale market on transmission level. It is possible via the exchange to import missing electricity or export excess supply. We assume a simple model for the agent controlling the exchange $q_{t} \in \mathbb{R}$. The agent optimizes on the price arbitrage between the price of the market platform $p_{t}^{\star}$ and the price or cost of electricity at the connected market given by the parameter $c_{t}^{q} \in \mathbb{R}$. The price arbitrage is defined by:

$$
g\left(q_{t}\right)=p_{t}^{\star} q_{t}-c_{t}^{q} q_{t} .
$$

The local MO solves the following optimization problem:

$$
\max _{q_{t}} g\left(q_{t}\right) .
$$

We let $q_{t}$ be the import (or export) of energy for the community, with perceived revenues $g\left(q_{t}\right)$. We adopt the following convention:

$-q_{t} \leq 0\left(\mu_{t}=1\right)$, there is an energy lack in the community and the local MO buys the missing quantity to the grid or to another local energy community. 
$-q_{t}>0\left(\mu_{t}<1\right)$, there is an energy excess in the community and the local MO sells the excess quantity to the grid or to another local energy community.

In order to justify an export of energy $\left(q_{t}>0\right)$, the price on the platform must be at least as high as the price of the exchange $\left(p_{t}^{\star} \geq c_{t}^{q}\right)$. The only possible case is that the price of the exchange is below the cost of the prosumers for which they are willing to share part of their generation. As such, the case might also be prevented by the setting of the price floor $p$.

\subsection{Designing a Trust-Based Mechanism}

Consumers and prosumers interact through a sharing platform. The platform operated by the local MO, matches the prosumers and the consumers ${ }^{3}$, and sets a clearing price. At each repetition $\nu \in \mathbb{N}^{*}$ of the game, the platform clearing price profile defined over $T_{C}$ consecutive time periods, $\left(p_{t}^{\star}\right)_{t=(\nu-1) T_{C}+1, \ldots, \nu T_{C}}$, is determined as solution of an optimal exchange problem [8]. Formally, as will be detailed later on, it is computed as a consensus variable.

After $\nu$ repetitions of $T_{C}$ consecutive time periods, a reputation index $R_{\nu}^{n}$ and an ancienty index $A_{\nu}^{n}$ are computed and associated to each node $n \in \mathcal{N}$. Based on these indexes, a miner node is selected in exchange of a mining fee. In practice, nodes in the system compete to solve the complex mathematical program (puzzle) necessary to validate the last block. Reputation indexes are introduced to build decentralized trust-based mechanisms and prevent the emergence of (large-scale) coalitions of nodes with Byzantine behaviors, which would attack the system [5]. In the context of our paper, we can imagine that consumer nodes group together to decrease artificially their aggregated demand (target demands being quite different from one consumer to another, one excess buying from one consumer can compensate a lack of buying from another consumer) and then make the market clearing price decrease, therefore potentially inducing supplyshortages. In Vangulick et al., the reputation index is a linear function of the quantity of energy broadcast previously by the node to mine blocks, the age of the last mined block and a trust index [13]. Furthermore, only consumer nodes can be selected as miners and the selection is made based on a random rule in which the node reputation index is weighted by a random uniform variable. We see no obvious reason to restrict the miner selection to consumer nodes only and define a selection rule less conservative than the one introduced in [13]. Our miner selection rule is based on a fixed-share exponentially-weighted average density function, which is far less energy-greedy than classical PoW methods used in Bitcoin, and less arbitrar than PoS methods used in Ethereum.

The game takes place over $\nu T_{C}$ consecutive time periods (or, alternatively, is repeated $\nu$ times), taking as input the demand and production schedules of consumers and prosumers. From $(\nu-1) T_{C}+1$ to $\nu T_{C}$, the game timing can be described as follows:

\footnotetext{
3 The matching process itself is out of the scope of the current paper and, as such, will not be detailed here.
} 
(i) Miner Selection A miner node $m_{\nu}^{\star}$ is selected based on his reputation index $\left(R_{\nu}^{n}\right)$ and how often he has not been selected in the past, that we will call ancienty $\left(A_{\nu}^{n}\right)$, following a fixed-share exponentially-weighted average density function, to guarantee the balance between exploration (of new nodes) and exploitation of other nodes having good reputation. Formally, for any $n \in \mathcal{N}$, the probability that node $n$ is selected as a miner is:

$$
\begin{aligned}
\mathbb{P}_{n, \nu}: & =(1-\gamma) \underbrace{\frac{1-\exp \left(-\zeta R_{\nu}^{n}\right)}{\sum_{n^{\prime} \in \mathcal{N}}\left(1-\exp \left(-\zeta R_{\nu}^{n^{\prime}}\right)\right)}}_{\text {exploitation }}+\gamma \underbrace{\frac{1-\exp \left(-\zeta A_{\nu}^{n}\right)}{\sum_{n^{\prime} \in \mathcal{N}}\left(1-\exp \left(-\zeta A_{\nu}^{n^{\prime}}\right)\right)},}_{\text {exploration }} \\
& \forall \nu \in \mathbb{N}^{*}, \\
\mathbb{P}_{n, 0}:=\frac{1}{N} &
\end{aligned}
$$

where $\gamma \geq 0$ is a parameter characterizing the trade-off between exploration and exploitation in the miner selection process and $\zeta$ determines the growth rate of the selection probability. The miner node receives a mining fee $\sum_{t=(\nu-1) T_{C}+1}^{\nu T_{C}} \Phi_{t}^{m m_{\nu}^{\star}}$, and the utility function of the nodes are updated as follows: $\Pi_{C, \nu}^{\star}:=\left[\sum_{t=(\nu-1) T_{C}+1}^{\nu T_{C}} \Pi_{C}\left(y_{t}^{C \star}\right)+\Phi_{t}^{C} \mathbf{1}_{m_{\nu}^{\star}=C}\right]$, and $\Pi_{P, \nu}^{\star}:=\left[\sum_{t=(\nu-1) T_{C}+1}^{\nu T_{C}} \Pi_{P}\left(x_{t}^{P \star}, \boldsymbol{y}_{t}^{\star}, \boldsymbol{s}_{t}^{\star}\right)+\Phi_{t}^{P} \mathbf{1}_{m_{\nu}^{\star}=P}\right]$.

\section{(ii) Consumer-Prosumer Interactions}

- The local MO computes the clearing price profile $\left(p_{t}^{\star}\right)_{t=(\nu-1) T_{C}+1, \ldots, \nu T_{C}}$ and sends it to the consumers and prosumers.

- Each consumer $C$ computes the demand schedule $\left(y_{t}^{C}\left(p_{t}^{\star}\right)\right)_{t=(\nu-1) T_{C}+1, \ldots, \nu T_{C}}$ that maximize his utility. Similarly, each prosumer $P$ computes her consumption schedule $\left(x_{t}^{P}\left(p_{t}^{\star}\right)\right)_{t=(\nu-1) T_{C}+1, \ldots, \nu T_{C}}$ from self-production and the quantity she wants to share on the platform $\left(s_{t}^{P}\left(p_{t}^{\star}\right)\right)_{t=(\nu-1) T_{C}+1, \ldots, \nu T_{C}}$.

(iii) Reputation Update A consensus-based algorithm is run by the platform for a finite positive number $n_{\text {iter }}$ of consecutive iterations, reputation index of the nodes are updated based on the divergences between their schedules and algorithm output, leading to the following rule for consumer $C \in \mathcal{C}$

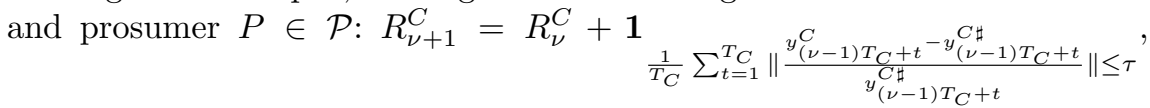
$R_{\nu+1}^{P}=R_{\nu}^{P}+\mathbf{1}_{\frac{1}{T_{C}} \sum_{t=1}^{T_{C}}\left\|\frac{x_{(\nu-1) T_{C}+t}^{P}-x_{(\nu-1) T_{C}+t}^{\sharp}}{x_{(\nu-1) T_{C}+t}^{\sharp}}\right\| \leq \tau}$. Variable $A_{\nu}^{n}$, called ancienty, captures how often node $n$ has not been selected as a miner until time period $\nu T_{C}$, it is updated according to the following rule: $A_{\nu+1}^{n}=$ $A_{\nu}^{n}+\mathbf{1}_{m_{\nu}^{\star} \neq n}, \forall n \in \mathcal{N}$.

Convergence of the Trust-Based Mechanism Consider the reputation (resp. ancienty) in the node selection probability. We let the gain function $h_{\nu}(n)$ 
be the indicator function in the reputation (ancienty) update and define the external regret as $\mathcal{R}\left(\mathbf{m}^{\star}, n_{\text {iter }}\right):=\sum_{\nu=1}^{n_{\text {iter }}}\left(-h\left(m_{\nu}^{\star}\right)\right)-\min _{n \in \mathcal{N}}\left(-h_{\nu}(n)\right)$. If the rate is chosen so that $\zeta=\sqrt{\frac{8 \ln (N)}{n_{\text {iter }}}}$, then $\lim _{n_{\text {iter }} \rightarrow \infty} \frac{1}{n_{\text {iter }}} \mathcal{R}\left(\mathbf{m}^{\star}, n_{\text {iter }}\right)=0$ with probability 1 as well-known in the weighted average forecasting literature. For $n_{\text {iter }}$ large enough, depending on the value of $\left.\left.\gamma \in\right] 0 ; 1\right]$, regret-minimizing nodes satisfying one or a combination of both criteria will be selected as miners.

Suppose Proposition 3 holds in the rest of the paper. This seems a reasonable assumption, as it is also observed in practice [3]. Assuming that the quantity $q_{t}$ on the local energy market at each time period is known, the market clearing writes down as a concave optimization problem, that can be interpreted as an optimal exchange problem [1], [8]:

$$
\begin{array}{ll}
\max _{\boldsymbol{y}, \boldsymbol{x}, \boldsymbol{s}} & \sum_{t=(\nu-1) T_{C}+1}^{\nu T_{C}}\left\{\sum_{C \in \mathcal{C}} \Pi_{C}\left(y_{t}^{C}\right)+\sum_{P \in \mathcal{P}} \Pi_{P}\left(x_{t}^{P}, s_{t}^{P}\right)+g\left(q_{t}\right)\right\}, \\
\text { s.t. } & x_{t}^{P}+s_{t}^{P} \leq \kappa^{P}, \forall P \in \mathcal{P}, \forall t, \\
& 0 \leq x_{t}^{P}, s_{t}^{P}, \forall P \in \mathcal{P}, \forall t, \\
& 0 \leq y_{t}^{C} \leq \kappa^{C}, \forall C \in \mathcal{C}, \forall t .
\end{array}
$$

\section{Solving the Platform Problem Using ADMM}

In the platform problem, the local MO determines the clearing price $\boldsymbol{p}_{t}^{\star}$ which maximizes the social welfare, under limited information exchange between the nodes. To apply decentralized optimization, we reformulate the platform optimization problem (16)-(19) by observing that the objective function can be decomposed onto the consumers and prosumers' decision variables.

\subsection{Reformulation as a Consensus Problem}

We set $\tilde{g}\left(q_{t}\right)=-c_{t}^{q} q_{t}$. The platform optimization problem can be formulated as follows:

$$
\begin{array}{ll}
\max _{\boldsymbol{y}, \boldsymbol{x}, \boldsymbol{s}} & \sum_{t=(\nu-1) T_{C}+1}^{\nu T_{C}}\left\{\sum_{C \in \mathcal{C}} \tilde{U}_{C}\left(y_{t}^{C}\right)+\sum_{P \in \mathcal{P}}\left[U_{P}\left(x_{t}^{P}\right)-c_{P}\left(x_{t}^{P}+s_{t}^{P}\right)\right]+\tilde{g}\left(q_{t}\right)\right\}, \\
\text { s.t. } & \sum_{C \in \mathcal{C}} y_{t}^{C}-\sum_{P \in \mathcal{P}} s_{t}^{P}+q_{t}=0, \forall t, \quad\left(p_{t}^{\star}\right) \\
& x_{t}^{P}+s_{t}^{P} \leq \kappa^{P}, \forall P \in \mathcal{P}, \forall t \\
& 0 \leq x_{t}^{P}, s_{t}^{P}, \forall P \in \mathcal{P}, \forall t \\
& 0 \leq y_{t}^{C} \leq \kappa^{C}, \forall C \in \mathcal{C}, \forall t .
\end{array}
$$

The platform problem can be solved in three different ways, that are pictured in Fig. 2: 
(a) Joint optimization of (20)-(24). Notice that this requires that the local MO has full information on consumers and prosumers' utilities.

(b) Mixed Complementarity Problem (MCP) reformulation solved as a squared system made of all the first-order stationarity conditions derived in Section 2. Notice that his requires that the local MO has full information on consumers and prosumers' preferences.

(c) Iteratively, with limited private information exchanges between the nodes.

We focus on approach (c) in this section. To determine the platform clearing price with limited exchange of information between the nodes, we resort to use an algorithmic approach. Our key technical tool is an optimization technique known as the ADMM [1], [4], [10]. Applying ADMM to our problem, a concave objective function of the form $\sum_{t=(\nu-1) T_{C}+1}^{\nu T_{C}}\left(\sum_{P \in \mathcal{P}} f_{P}\left(\boldsymbol{X}_{t}^{P}\right)+\sum_{C \in \mathcal{C}} f_{C}\left(\boldsymbol{Y}_{t}^{C}\right)\right)$ is maximized subject to some constraints by performing alternating individual optimizations over $f_{C}($.$) and f_{P}($.$) . While it was originally introduced to achieve$ faster convergence [1], it was observed in [10] that when the functions $f_{C}($. and $f_{P}($.$) are private information belonging to consumer C$ and prosumer $P$, ADMM has the additional advantage of sharing only a small amount of private information between the two parties.

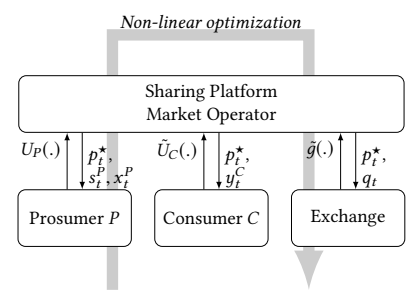

(a) Joint Optimization

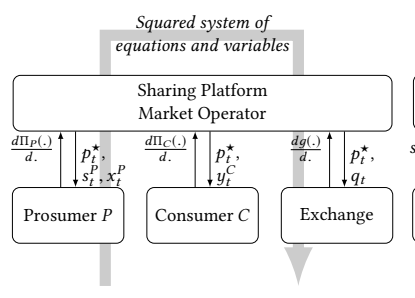

(b) MCP reformulation

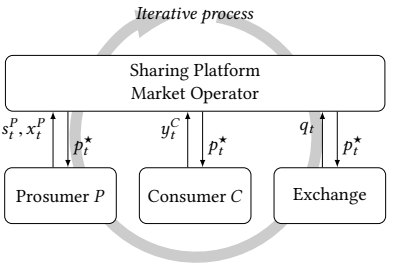

(c) ADMM process

Fig. 2: The three approaches and necessary information exchanges for the local market operator to clear the platform market.

In the general formulation, agents are trying to jointly solve the generic concave optimization problem:

$$
\begin{aligned}
\max _{\boldsymbol{X}, \boldsymbol{Y}, \boldsymbol{Z}} & \sum_{t=(\nu-1) T_{C}+1}^{\nu T_{C}}\left(\sum_{P \in \mathcal{P}} f_{P}\left(\boldsymbol{X}_{t}^{P}\right)+\sum_{C \in \mathcal{C}} f_{C}\left(\boldsymbol{Y}_{t}^{C}\right)+h\left(\boldsymbol{Z}_{t}\right)\right), \\
\text { s.t. } & \boldsymbol{A}_{P}\left(\begin{array}{c}
\boldsymbol{X}_{t}^{P} \\
\boldsymbol{Z}_{t}
\end{array}\right) \leq \boldsymbol{b}_{P}, \forall P \in \mathcal{P}, \forall t \\
& \boldsymbol{A}_{C}\left(\begin{array}{c}
\boldsymbol{Y}_{t}^{C} \\
\boldsymbol{Z}_{t}
\end{array}\right) \leq \boldsymbol{b}_{C}, \forall C \in \mathcal{C}, \forall t \\
& \boldsymbol{B} \boldsymbol{Z}_{t} \leq \boldsymbol{b}, \forall t .
\end{aligned}
$$


By identification with the relaxed optimal exchange problem (16)-(19), we set: $\boldsymbol{X}_{t}^{P}:=\left(x_{t}^{P}, s_{t}^{P}\right), \boldsymbol{Y}_{t}^{C}:=y_{t}^{C}$, and $\boldsymbol{Z}_{t}:=p_{t}^{\star}$. Regarding the objective functions, we set: $f_{P}\left(\boldsymbol{X}_{t}^{P}\right)=\Pi_{P}\left(x_{t}^{P}, s_{t}^{P}\right), f_{C}\left(\boldsymbol{Y}_{t}^{C}\right)=\Pi_{C}\left(y_{t}^{C}\right), h\left(\boldsymbol{Z}_{t}\right)=g\left(q_{t}\right)$.

The time periods being not linked, we decompose (25) over each time period $t$, so that the vectors constraints at $t$ take the form: $\boldsymbol{A}_{P}=\left(\begin{array}{ccc}1 & 1 & 0 \\ -1 & 0 & 0 \\ 0 & -1 & 0\end{array}\right), \boldsymbol{b}_{P}=$ $\left(\begin{array}{c}\kappa^{P} \\ 0 \\ 0\end{array}\right), \boldsymbol{A}_{C}=\left(\begin{array}{cc}1 & 0 \\ -1 & 0\end{array}\right), \boldsymbol{b}_{C}=\left(\begin{array}{c}\kappa^{C} \\ 0\end{array}\right)$, and $\mathbf{B}=(1-1), \mathbf{b}=(\bar{p}-\underline{p})$.

In our case, $f_{n}(),. \boldsymbol{b}_{n}$ constitute the information privately held by node $n \in$ $\mathcal{N}$, whereas $h(),. \boldsymbol{B}$ and $\boldsymbol{b}$ are known to all nodes. We want to solve this concave optimization problem so that in the optimum, $\boldsymbol{X}^{P}\left(\operatorname{resp} . \boldsymbol{Y}^{C}\right)$ is private output known only by prosumer $P$ (resp. consumer $C$ ), but $\boldsymbol{Z}$ may be known to all. ADMM uses an iterative process to solve the optimization problem and only shares the iterative updates of the shared variable $\boldsymbol{Z}$.

To make the link with the classical consensus-based approach [1], [10], we consider a slightly different concave optimization problem:

$$
\begin{aligned}
\max _{\boldsymbol{u}, \boldsymbol{v}} & \sum_{t=(\nu-1) T_{C}+1}^{\nu T_{C}}\left(F\left(\boldsymbol{u}_{t}\right)+G\left(\boldsymbol{v}_{t}\right)\right), \\
\text { s.t. } & \boldsymbol{B}_{1} \boldsymbol{u}_{t} \leq \boldsymbol{d}_{1}, \forall t \\
& \boldsymbol{B}_{2} \boldsymbol{v}_{t} \leq \boldsymbol{d}_{2}, \forall t \\
& \boldsymbol{u}_{t}=\boldsymbol{v}_{t}, \forall t
\end{aligned}
$$

Proposition 4. Problem (26) is a special case of Problem (25).

Proof of Proposition 4. We can construct Problem (26) where the variables $\boldsymbol{u}_{t}$ and $\boldsymbol{v}_{t}$ both represent an independent copy of $\left(\left(X_{t}^{P}\right)_{P \in \mathcal{P}},\left(y_{t}^{C}\right)_{C \in \mathcal{C}}, Z_{t}\right)$. Formally, let the objective function $F($.$) and G($.$) be defined as follows: F\left(\boldsymbol{u}_{t}\right)=$ $\sum_{P \in \mathcal{P}} f_{P}\left(\boldsymbol{X}_{t}^{P}\right)+\frac{h\left(\boldsymbol{Z}_{t}\right)}{2}, G\left(\boldsymbol{v}_{t}\right)=\sum_{C \in \mathcal{C}} f_{C}\left(\boldsymbol{Y}_{t}^{C}\right)+\frac{h\left(\boldsymbol{Z}_{t}\right)}{2}$. It is easy to see that $\boldsymbol{B}_{1}$ and $\boldsymbol{d}_{1}$ can be created by inserting zeros in appropriate places such that the constraint set $\boldsymbol{B}_{1} \boldsymbol{u}_{t} \leq \boldsymbol{d}_{1}$ reduces to the union of $\boldsymbol{A}_{P}\left(\boldsymbol{X}_{t}^{P} \boldsymbol{Z}_{t}\right)^{T} \leq \boldsymbol{b}_{P}, \forall P \in \mathcal{P}$ and $\boldsymbol{B} \boldsymbol{Z}_{t} \leq \boldsymbol{b} . \boldsymbol{B}_{2}$ and $\boldsymbol{d}_{2}$ can be generated following the same way, e.g., by inserting zeros in appropriate places such that the constraint set $\boldsymbol{B}_{2} \boldsymbol{v}_{t} \leq \boldsymbol{d}_{2}$ reduces to the union of $\boldsymbol{A}_{C}\left(\boldsymbol{Y}_{t}^{C} \boldsymbol{Z}_{t}\right)^{T} \leq \boldsymbol{b}_{C}, \forall C \in \mathcal{C}$ and $\boldsymbol{B} \boldsymbol{Z}_{t} \leq \boldsymbol{b}$. This completes the construction of Problem (26).

\subsection{Updating Rules, Privacy Preservation and Stopping Criteria}

We define $\mathcal{F} \mathcal{S}(\boldsymbol{u}):=\left\{\boldsymbol{u} \mid \boldsymbol{B}_{1} \boldsymbol{u} \leq \boldsymbol{d}_{1}\right\}$ as the feasibility set of $\boldsymbol{u}$ and $\mathcal{F} \mathcal{S}(\boldsymbol{v}):=$ $\left\{\boldsymbol{v} \mid \boldsymbol{B}_{2} \boldsymbol{v} \leq \boldsymbol{d}_{2}\right\}$ as the feasibility set of $\boldsymbol{v}$. Optimization problem (26) can be decomposed in time. So, ADMM solves Problem (26) in an iterative fashion 
[10], where for each time period $t \in\left\{(\nu-1) T_{C}+1, \ldots, \nu T_{C}\right\}$, each iteration $k$ has three steps as described below:

$$
\begin{aligned}
& \boldsymbol{u}_{t}^{k+1} \in \arg \min _{\boldsymbol{u} \in \mathcal{F}(\boldsymbol{u})}\left\{-F(\boldsymbol{u})+\boldsymbol{\lambda}^{k T} \boldsymbol{u}+\frac{\rho}{2}\left\|\boldsymbol{u}-\boldsymbol{v}_{t}^{k}\right\|^{2}\right\}, \\
& \boldsymbol{v}_{t}^{k+1} \in \arg \min _{\boldsymbol{v} \in \mathcal{F} \mathcal{S}(\boldsymbol{v})}\left\{-G(\boldsymbol{v})+\boldsymbol{\lambda}^{k T} \boldsymbol{v}+\frac{\rho}{2}\left\|\boldsymbol{u}_{t}^{k+1}-\boldsymbol{v}\right\|^{2}\right\}, \\
& \boldsymbol{\lambda}_{t}^{k+1}=\boldsymbol{\lambda}_{t}^{k}+\rho\left\|\boldsymbol{u}_{t}^{k+1}-\boldsymbol{v}_{t}^{k+1}\right\|^{2} .
\end{aligned}
$$

Note that the consumer and prosumer optimization problems being separable, first step can be solved independently by each prosumer $P \in \mathcal{P}$; second step can be solved independently by each consumer $C \in \mathcal{C}$; while last step is computed by the local MO.

The update steps of the consensus-based ADMM algorithm violate the output privacy requirements because $\boldsymbol{u}_{t}$ (resp. $\boldsymbol{v}_{t}$ ), which is revealed to the consumers (resp. prosumers), contains a copy of the private output variables of all the other agents. However, the key point to observe is that the optimization problem in each step can be decomposed into components that depend on different individual variables of $\boldsymbol{u}_{t}$ (resp. $\boldsymbol{v}_{t}$ ). Therefore, the set of components in optimization steps of consumers that depend on the private output of the other agents can effectively be removed from the objective function, and at the same time, the feasible region $\mathcal{F S}($.$) can be reduced to the feasible region over \boldsymbol{X}_{t}^{P}, Z_{t}$ for any prosumer $P$ (resp. $\boldsymbol{Y}_{t}^{C}, Z_{t}$ for any consumer $C$ ). Hence, the optimization can be carried out in a way that each agent is only revealed her final value $\boldsymbol{X}_{t}^{P}\left(\boldsymbol{Y}_{t}^{C}\right)$ and $Z_{t}$. Hence, the output privacy is also preserved by the consensus-based ADMM algorithm.

ADMM iterates satisfy the following:

- objective convergence $\sum_{t=(\nu-1) T_{C}+1}^{\nu T_{C}}\left(F\left(\boldsymbol{u}_{t}^{k}\right)+G\left(\boldsymbol{v}_{t}^{k}\right)\right) \rightarrow_{k} F^{\star}+G^{\star}$, where $F^{\nu, \star}+G^{\nu, \star}$ are the optimal value of the optimization problem (26) with $T_{C}$ time period look-ahead at $\nu$-th repetition of the platform game.

- dual variable convergence $\lambda^{k} \rightarrow_{k} p_{t}^{\star}$.

- residual convergence $r^{k}:=\left\|u^{k}-u^{\star}\right\|+\left\|v^{k}-v^{\star}\right\| \rightarrow_{k} 0$, where $u^{\star}, v^{\star}$ contain the optimum values for the prosumers and consumers as described in Propositions 1 and 2.

\section{Case Study}

In this section, an illustrative case study is presented. The objective of the case study is twofold. Firstly, to exhibit the parts of the equilibrium model and the algorithm to solve it (subsection 4.1). Secondly, to present the miner selection process and its dynamics (subsection 4.2). Note that subsection 4.1 focuses on the equilibrium and the impact of consumer preferences with regard to additional characteristics, while subsection 4.2 highlights results of the introduced ADMM algorithm on e.g., the number of required iterations, the dynamics of the miner selection process, and the impact of the threshold parameter $\tau$. 
In the case study a consumer $(C)$ interacts with two prosumers $\left(P_{1}\right.$ and $P_{2}$ ) via a platform (acting as the intermediate). For his demand, $C$ chooses between two product characteristics: locality (Loc) and RES-based (RES). The preferences of $C$ serve to match his demand with the supply characteristics of $P_{1}$ and $P_{2}$. Tables 1 and 2 show the values of the parameters to be used throughout the case study, for the consumer and prosumer models, respectively.

Table 1: Input parameter of consumer for case study.

\begin{tabular}{|c|c|c|c|}
\hline Consumer $C$ & & Prosumer 1 Prosumer 2 & |0 Loc RES \\
\hline$y_{t}^{C \sharp} 35$ & $\alpha_{\mathrm{LOC}}^{C P}$ & 0.3 & $\left|\xi^{C}\right|-0.20 .8$ \\
\hline$\kappa^{C} 80$ & $\alpha_{\mathrm{RES}}^{C P}$ & 0.3 & 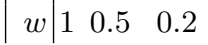 \\
\hline
\end{tabular}

Table 2: Input parameter of prosumers for case study.

\begin{tabular}{c|cc|ccc}
\hline Prosumer $P$ & $x_{t}^{P \sharp}$ & $\kappa^{P}$ & $c_{P 2}$ & $c_{P 1}$ & $c_{P 0}$ \\
\hline Prosumer 1 & 40 & 60 & 1 & 20 & 0 \\
Prosumer 2 & 40 & 60 & 0.1 & 10 & 0 \\
\hline
\end{tabular}

\subsection{Impact of Consumer Preferences on Locality and RES-Based Generation}

We notice a linear relation between the consumer demand and the market clearing price. This observation coincides with the results that we analytically derived in Proposition 1. We also observe that the market price (and then, the demand) is maximum in case the consumer is indifferent between the two prosumers for his RES-based demand. That is, the location of the prosumer is irrelevant when looking to fulfill his RES preference. Furthermore, preference regarding one prosumer seems to have a limited impact on his demand. We conclude that the definition of the consumer matching preferences for his RES-based demand have a direct impact on his relative consumption, and, on his utility which is maximized in case where the consumer is indifferent between both prosumers. Furthermore, to maximize his utility the consumer can have strong preference regarding RES-based generation but limited preference regarding locality. In general, the consumer should keep his preference regarding locality moderate to maximize his utility.

\subsection{Miner Selection: Dynamics and Threshold}

In Fig. 3, we represent the error distribution of three nodes, based on market results for 24 market clearings, i.e., for each hour of the day. The error is defined 


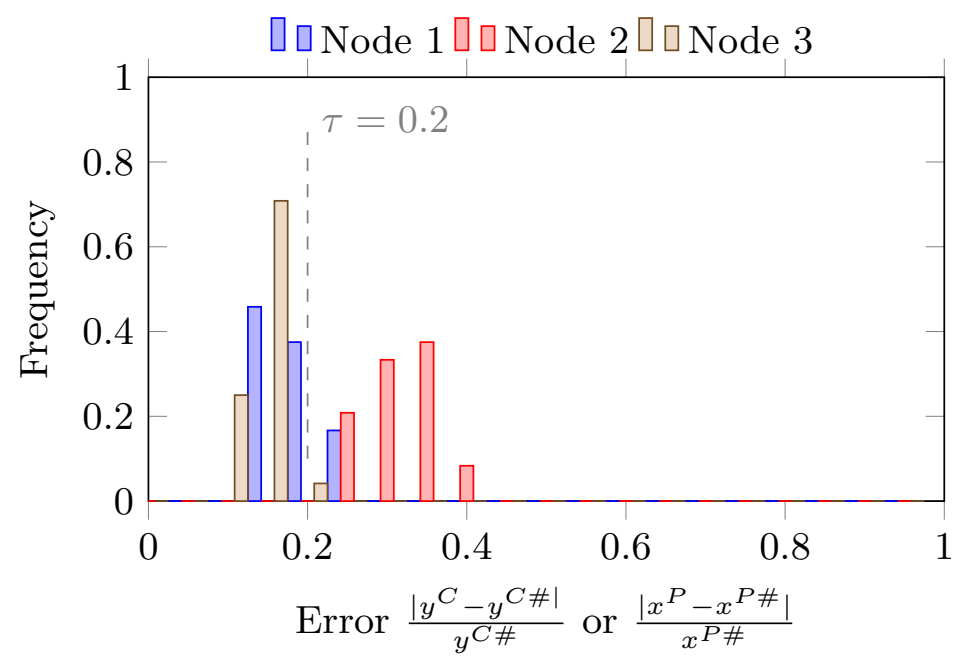

Fig. 3: Frequency of error terms of nodes based on target value (used during update of reputation)

as the normalized difference between the demand (auto-consumption) obtained as outcome of the consensus algorithm and the target value, and used to update the node reputation. Based on these equations, the impact of the error term also depends on the chosen threshold $\tau$. We observe that for values of $\tau>0.2$, node 2 generates the largest errors. As a result, if $\tau \leq 0.2$, node 2 reputation will remain unchanged. For $\tau \leq 0.1$ or $\tau \geq 0.4$, all the nodes keep their reputation unchanged. Bear in mind that the reputation is updated (i.e., increases by 1) if the node's error is below the threshold $\tau$. Nodes 1 and 3 have lower errors than node 2, with high frequencies for low errors at one node or the other. Meaning that with a $\tau=0.15$, node 1 has the largest reputation, while a bigger threshold e.g., $\tau=0.18$ gives node 3 a larger reputation.

The proposed selection process accounts for the exploration and exploitation of miners. Fig. 5 illustrates the dynamics of the selection process in terms of $\gamma$; parameter that controls for the trade-off between exploration of new miners and the exploitation of miners with high reputation.

If we assume an equal weighting of the exploitation and exploration probabilities, i.e., $\gamma=\frac{1}{2}$ (Fig. 5a), we observe the following:

- node 2's reputation worsened, in respect to the others' nodes, with the number of market clearings. The exploration term is not able to compensate for the low reputation.

- nodes 1 and 3 show a higher selection probability than node 2 . This probability oscillates during the first clearings and settles around a value of 0.2 . Note that both nodes show values that are close enough to be considered as identical. This, for more than 15 repetitions of the game. 


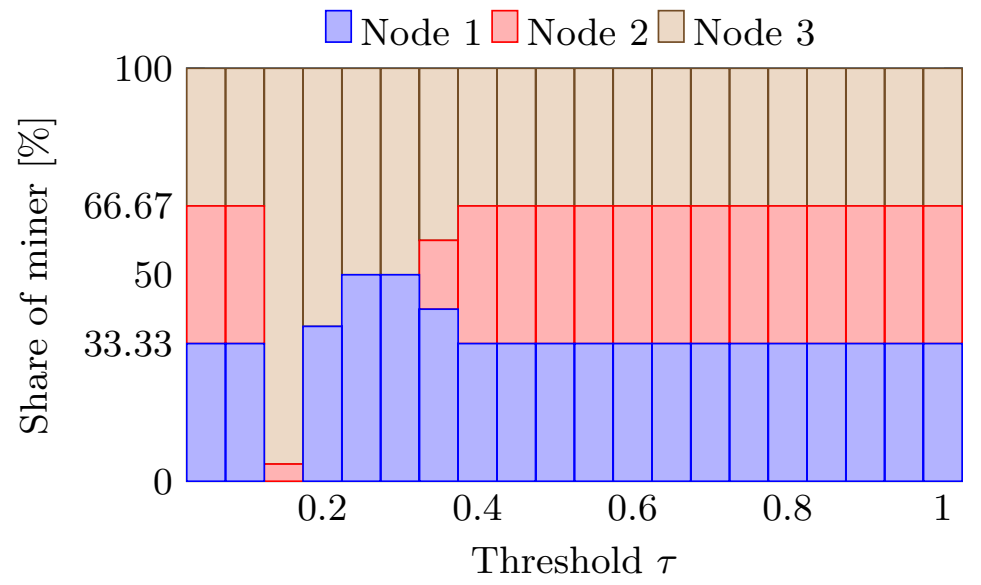

Fig. 4: Miner selection for 24 market clearings with changing values for $\tau$

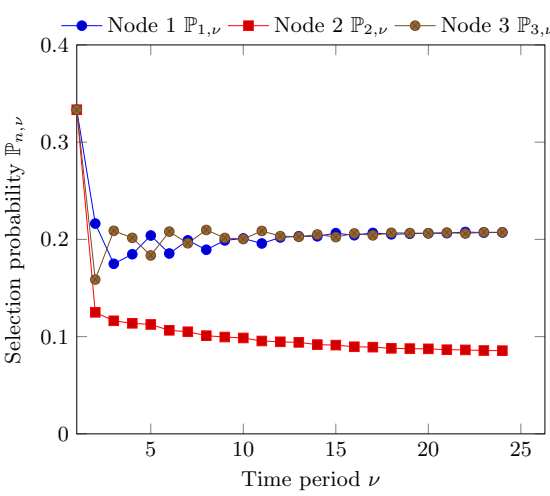

(a) $\gamma=0.5$

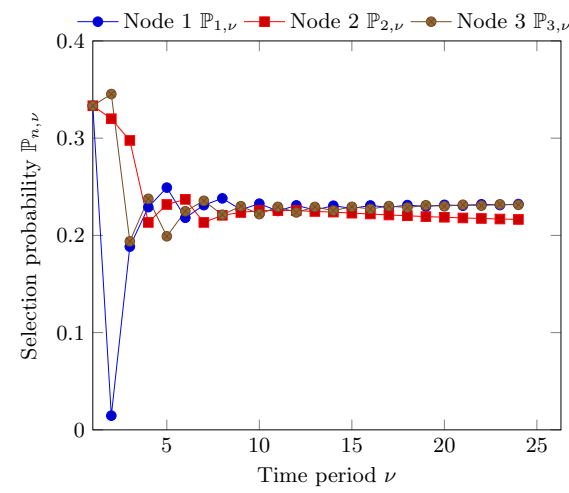

(b) $\gamma=0.8$

Fig. 5: Dynamics of miner selection process (Parameter $\gamma$ to control trade-off between exploration and exploitation. $\tau=0.2$ ) 
When a higher emphasis is given to the exploration of new miners, that is $\gamma=0.8$, we observe a quite different picture (Fig.5b). Node 2 gets selected as a miner in the beginning, and its chance to get selected remains at a relatively high level compared to the others. Forcing the process to look for new miners increases substantially the chances of poor performing nodes to become the miner. This, even if the reputation is low (and never updates) and the threshold $\tau$ is such that with a $\gamma=\frac{1}{2}$ (i.e., no favoritism for exploitation or exploration) this node would never be selected as the miner (see Fig. 4).

As such, a $\gamma=0.8$ assigns a higher probability to select node 2 as the miner during all clearings than assuming $\gamma=\frac{1}{2}$. Consequently, $\gamma$ provides a valuable tuning parameter to control the miner selection process.

\section{Conclusions}

In this paper, we consider consumers and prosumers who interact via a platform. On the one hand, consumers specify their target demand and optimize their demand to the platform in order to find a trade-off between maximizing their usage benefit and minimizing the cost they pay to the platform. On the other hand, prosumers need to determine the amount of generated energy they selfconsume and the quantity they share on the platform. Our study introduces product differentiation and consumers's preferences, namely locality and RESbased generation. These preferences are used to match the prosumers generation characteristics. We introduce the probability for a prosumer to be matched to a consumer. In case the consumer demand is larger than the prosumer supply, the matching problem can be decomposed in decoupled optimization problems, that we solve analytically. In case of an excess of supply compared to the demand on the platform, the consumers and prosumers problems remain coupled through the matching probability, giving rise to a non-cooperative game. We provide analytical conditions for the existence and uniqueness of a Nash equilibrium.

We prove the existence of a market clearing price cap below which supplyshortages occur on the platform. Under this assumption (also observed on Didi Chuxing, the largest ridesharing platform in China), we implement ADMM reformulated as a consensus problem, to solve this specific platform issue. A trustbased mechanism is implemented on top of it, to select at each repetition of the game (clearings) the node which validates the transactions (e.g., demand, self-consumption and shared production from the prosumers), that is the node that acts as the miner. The miner node selection is made according to a density function capturing the trade-off between exploitation of nodes with good reputation and exploration of new nodes. The goal is to prevent that the nodes deviate too much from their target schedules, forming coalitions that could work independently of the platform.

Our case study quantifies the impact of consumers' preferences for the matching with prosumers and assess the dynamics of the miner selection process for three nodes. The case study shows that although product differentiation could in theory drive the consumers decision on how to supply his/her demand (based 
on his/her preferences), in practice the decision's main driver is the price it pays for the product (in this case, energy). In addition, we observed that a tunning parameter that captures the trade-off between exploring for new miners and exploiting nodes with good reputation is relevant for the control of the miner selection process.

\section{References}

1. Boyd, S., Parikh, N., Chu, E., Peleato, B., Eckstein, J.: Distributed optimization and statistical learning via the alternating direction method of multipliers. Foundations and Trends in Machine Learning (2018)

2. Fagnani, F., Zampieri, S.: Randomized consensus algorithms over large scale networks. IEEE Journal on Selected Areas in Communications 26 (4), 634-649 (2018)

3. Fang, Z., Huang, L., Wierman, A.: Prices and Subsidies in the Sharing Economy. In 26th International Conference on World Wide Web - WWW'17, pp. 53-62. ACM Press (2017). doi.10.1145/3038912.3052564

4. Höschle, H., Le Cadre, H., Smeers, Y., Papavasiliou, A., Belmans, R.: An ADMMbased Method for Computing Risk-Averse Equilibrium in Capacity Markets. IEEE Transactions on Power Systems, 33 (5), 4819-4830 (2018)

5. Lamport, L., Shostak, R., Pease, M.: The Byzantine Generals Problem. ACM Transactions on Programming Languages and Systems 4 (3), 382-401 (1982)

6. Le Cadre, H.: On the efficiency of local electricity markets under decentralized and centralized designs: a multi-leader Stackelberg game analysis. Central European Journal of Operations Research (CEJOR), in press (2018) doi.10.1007/ s10100-018-0521-3

7. Le Cadre, H., Mezghani, I., Papavasiliou, A.: A Game-Theoretic Analysis of Transmission-Distribution System Operator Coordination. European Journal of Operational Research (EJOR), 274 (1), 317-339 (2019)

8. Moret, F., Pinson, P.: Energy Collectives: a Community and Fairness based Approach to Future Electricity Markets. IEEE Transactions on Power Systems, in press (2018). doi.10.1109/TPWRS.2018.2808961

9. Munsing, E., Mather, J., Moura, S., Bolckchains for decentralized optimization of energy resources in microgrid networks. In: International Conference on Control Technology and Applications, IEEE (2017). doi.10.1109/CCTA. 2017.8062773

10. Jiang, A. X., Procaccia, A. D., Qian, Y., Shah, N., Tambe, M.: Defender (mis)Coordination in Security Games via ADMM. In: IJCAI'13, pp. 220-226. ACM Press. (2018)

11. Szabo, N., Smart contracts: Building Blocks for Digital Markets. Extropy 16 (1996)

12. Tushar, W., Yuen, C., Mohsenian-Rad, H., Saha, T., Poor, V., Wood, K. L.: Transforming Energy Networks via Peer to Peer Energy Trading: Potential of Game Theoretic Approaches. IEEE Transactions on Signal Processing 247(935), 529-551 (2018)

13. Vangulick, D., Cornélusse, B., Ernst, D.: Blockchain for peer-to-peer energy exchanges: design and recommendations. In: PSCC conference, Dublin, Ireland (2018)

14. Wang, Y., Saad, W., Han, Z., Poor, V., Başar, B.: A Game-Theoretic Approach to Energy Trading in the Smart Grid. IEEE Transactions on Smart Grid 5 (935), 1439-1450 (2014)

15. Zhang, C., Wu, J., Long, C., Cheng, M.: Review of existing peer-to-peer energy trading projects. Energy Procedia 105, 2563-2568 (2016) 